\title{
Queueing Model for Medical Centers (A Case Study of Shehu Muhammad Kangiwa Medical Centre, Kaduna Polytechnic)
}

\author{
M.O. Oladejo and M.C. Aligwo \\ Department of Mathematics and Computer Science, Nigerian Defence Academy, Kaduna State, Nigeria \\ Department of Mathematics/Statistics/Computer science, Kaduna Polytechnic, Kaduna State, Nigeria.
}

\begin{abstract}
This paper examined the queueing situation in Shehu Mohammed Kangiwa Medical Center Kaduna Polytechnic. The complaints of patients on delays were corroborated by the 9minutes mean queue time and mean queue length of 3persons in the existing structure. A new multy -channel queueing model which yielded a mean queue time of Iminute and a mean queue length of Opersons was formulated. This multy-channel queueing model gave optimal results and is proposed for adoption.
\end{abstract}

Key words: Medical Center, Multy-Channel Queueing Model, Patients, Queue length, Queue time.

\section{Introduction}

The rise in the population of Nigeria translates into the increase in the demand for health care services. The Federal Government of Nigeria not oblivious of the adage that "A Healthy Nation Is A Wealthy Nation" established Federal Medical Centers In every state of the federation and other medical institutions and agencies such as National Institute for Trypanosomiasis research (NITR), National Drug Law Enforcement Agency (NDLEA), the National Health Insurance Scheme (NHIS), just to mention only a few. There are also health policies in place which make provisions for services like the National Immunization program, free treatment for T.B patients etc. Government policies also encourage the private sector who augments her efforts by the establishment of private hospitals.

The state governments are not left out. In fact Kaduna state government for instance has a well maintained hospital in each Local government area, with adequate supply of drugs. Pregnant women and children enjoy free health care services. All these efforts are to put a check against drug abuse, the peddling of injurious drugs, and to ensure that the citizenry is exposed to adequate health care delivery which is defined by [1] as the process by which an individual state of health is monitored, imbalances in the state and deviations from the desired state are detected and decisions are taken as to the application of available healthcare resources.

\subsection{Statement of Problem}

The health sector, in spite of all the efforts by the federal, state governments and the private sector to provide adequate health care services to the citizenry, is still entangled with some constraints. One of these constraints is the issue of queues (delay) in our hospitals resulting in a queueing problem, [2]. This problem impedes prompt delivery of health care services, a situation which could be disastrous as human life is involved and could lead to complications or even death.

\subsection{Literature Review}

A queue simply put is a place where customers wait before being served, [3], and they are formed when the current demand for a service exceeds the current capacity to provide the service, [4]. The problem of eliminating or reducing a queue could be very expensive as stated by [5]. But time they say is money, hence according to [6], time spent on queue is often costly therefore the dire need to solve the problem of queueing, and this in turn leads to queueing theory,

According to [7], queueing theory is the development of mathematical models for analysis, forecasting and optimal decision making of systems involving queues. The idea of queueing theory can be traced back to the classical work of A.K Erlang in 1900s, however the work of D.K Kendal in 1951 formed the basis for analytical calculations and the naming convention in queues being used today [8;9]

A schematic representation of the basic queueing process is given below as illustrated by [3] 
Customers queueing system

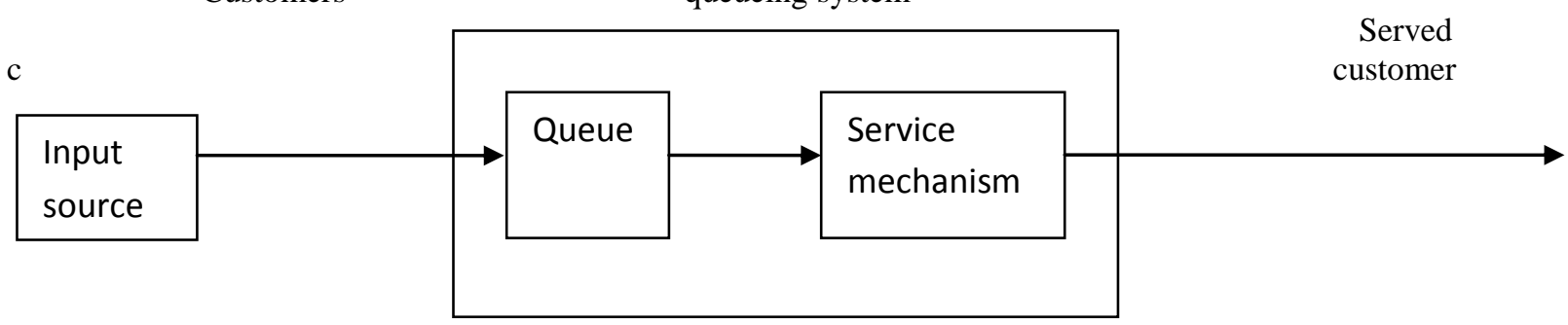

[10] and [3] explain that customers on arriving a queueing system would naturally join the queue. Occasionally, a customer is selected for service by a rule known as queue discipline. They are then attended to by a service facility called channel or server. A queueing system according to [6] involves a number of servers. One with one server is called a single-channel system while one with many servers is called a multiple -channel system. When service is completed the customer leaves the System.

\subsection{Objective of the Study}

The objectives of this study are:

i. Existing structure will be used to find values for queueing parameters by computation.

ii. Alternative model will be fashioned out and values of parameters compiled.

iii. To compare and contrast both models and provide the best model from values of the parameters.

\subsection{Significance of Study}

The result of the study will contribute immensely to:

i. Providing management with a tool to determine the number and or type of facilities to provide.

ii. Ensuring prompt attention to patients.

iii. Check avoidable complications or fatalities that may occur due to delays.

\section{Methodology}

Data were collected on Arrival time and service time on 350 patients out of those that consulted at the hospital during the period of this study, with exponential arrival rate and service time. They were now used to investigate the existing structure and compute new values for the queueing parameters. There after a model was structured to accommodate the main characteristics of the queueing system (hospital).

\subsection{Data Collection}

The source data was the Out-Patients Department. Data were collected from both primary and secondary sources between the hours of $8.00 \mathrm{am}$ to $10.00 \mathrm{am}$ in the morning for a period of ten days.

\subsection{The Existing Structure (M/M/3/Fcfs/ $/ \infty / \infty)$}

As at the time of this study, three (3) doctors were regularly attending to patients. Being a multychannel system, the following formulae were used for our calculations.

1. Service intensity which is the rate at which the service facility is utilized by the components of the system is given by

$\rho=\frac{\lambda}{\mathrm{K} \mu}$

where $\mathrm{K}$ is the number of channels and $\mathrm{K} \mu$ is the mean combined service rate for all the service stations.

2. The mean number of patients in the queue (queue length) is given by;

$\mathrm{Lq}=\frac{\mu \lambda\left(\frac{\lambda}{\mu}\right) \mathrm{P}_{\mathrm{O}}}{(\mathrm{K}-1) !(\mathrm{K} \mu-\lambda)^{2}}$

3. The mean number of patients in the system is

$\mathrm{L}=\mathrm{Lq}+\frac{\lambda}{\mu}$

4. The mean time spent in the queue (queue time) is

$\mathrm{Wq}=\frac{\mu\left(\frac{\lambda}{\mu}\right) \mathrm{P}_{\mathrm{O}}}{(\mathrm{K}-1) !(\mathrm{K} \mu-\lambda)^{2}}$

5. The mean time spent in the system is given by

$\mathrm{W}=\frac{\mu\left(\frac{\lambda}{\mu}\right) \mathrm{P}_{\mathrm{O}}}{(\mathrm{K}-1) !(\mathrm{K} \mu-\lambda)^{2}}+\frac{1}{\mu}$

6. The probability that there is no patient in the hospital is given by 
$\mathrm{P}_{0}=\left[\begin{array}{l}\mathrm{K}-1 \\ \sum \frac{1}{\mathrm{n} !}\left(\frac{\lambda}{\mu}\right)^{\mathrm{n}}+\left(\frac{\lambda}{\mu}\right)^{\mathrm{k}} \frac{1}{\mathrm{k} !(1-\rho)}\end{array}\right]^{-1}$

\section{Results And Discussion}

After analyzing the data to obtain values for the parameters, calculations and results were as follows:

\subsection{Computations with Existing Model}

Total number of patients $=350$

Number of channels (doctors) $\mathrm{K}=3$

Total arrival time $=1146$ minutes.

Total service time $=2764$ minutes.

Mean arrival rate $\lambda=\frac{\text { total no.of patients }}{\text { total arrival time }}=\frac{350}{1146}=0.3054$ minutes

Mean service rate $\mu=\frac{\text { total no.of patients }}{\text { total service time }}=\frac{350}{2764}=0.1266$ minutes

The ratio $\frac{\lambda}{\mu}=\frac{0.3054}{0.1266}=2.4123$

Service intensity $\rho=\frac{\lambda}{\mathrm{k} \mu}=\frac{0.3054}{3 \times 0.1266}=0.8045$

The probability of no patient in the hospital $\mathrm{P}_{0} \mathrm{By}(6)=0.0579$.

Now the queue length Lq by $(2)=2.8288 \approx 3$ patients

Time spent on queue $\mathrm{Wq}$ by $(4)=9.2703 \approx 9$ minutes

Mean number of patients in Hospital L by $(3)=5.2411 \approx 5$ patients

Mean time spent in the system $\mathrm{W}$ by $(5)=17.1692 \approx 17$ minutes

Results show that a patient spends an average of 9minutes in the queue and 17 minutes in the entire hospital.

It is obvious from the foregoing that patients spend so much time in the Hospital particularly in the queue waiting to consult a doctor. A situation such as this could lead to fatality, as the adage says "delay is dangerous" hence the need for a model to help eliminate or reduce to the barest minimum waiting time in particular.

\subsection{Investigation for a Workable Model}

\subsubsection{The Trial Model $\quad \mathrm{m} / \mathrm{m} / \mathbf{1} / \mathbf{f c f s} / \mathbf{1 5} / \infty$}

We commence work to formulate a workable model for the medical centre by grouping the patients into four (4) categories namely: Staff, Students, Gynaecology, Private

Each category was assigned a channel, hence we had four (4) channels .Though a multy-channel system, we adopted the principle of the $\mathrm{m} / \mathrm{m} / 1$ model and did our analysis in the system as if in a single channel system. Consequent upon this, we had four parallel queues one for each channel. The mean arrival rate, mean service rate, and service intensity were derived for each channel. Among others, the following formulae were also employed for our computations.

7. Probability of no patient in the hospital:

$\pi \mathrm{o}=\frac{1-\rho}{1-\rho^{c+1}}$

8. Mean number of patients in the system is given by:

$\mathrm{L}=\frac{\rho\left[1-(\mathrm{c}+1) \rho^{c}+\rho^{c+1}\right]}{\left(1-\rho^{c+1}\right)(1-\rho)}$

9. Mean number of patients on the queue:

$\mathrm{Lq}=\mathrm{L}-\frac{\lambda}{\mu}$

10. Mean time spent in the system:

$\mathrm{W}=\frac{\mathrm{L}}{\lambda\left(1-\pi_{c}\right)} \quad$ where $\pi \mathrm{c}=\rho \mathrm{c} \pi \mathrm{o}$

11. Mean time spent on queue is give by:

$\mathrm{Wq}=\frac{\mathrm{L}_{\mathrm{q}}}{\lambda\left(1-\pi_{c}\right)}$

\subsubsection{Computations with the Trial Model}

After analysis of data, computations and results in the respective categories are as follows: 
Category (A)

Channel capacity $\mathrm{C}=15$

Number of patients $=106$

Total inter-arrival time $=994 \mathrm{mins}$.

Total service time $=820 \mathrm{mins}$.

by (7), $\lambda=6.3984 / \mathrm{hr}$; by (8) $\mu=7.7561 / \mathrm{hr}$; by (9), $\rho=0.8250$.

\section{Category $(B)$}

Channel capacity $\mathrm{C}=15$

Total number of patients $=70$ minutes.

Total inter-arrival time $=1077$ minutes .

Total service time $=585$ minutes

By (7), $\lambda=3.8997 / \mathrm{hr}$; by (8), $\mu=7.1795 / \mathrm{hr}$; by (9), $\rho=0.5432$

\section{Category $(C)$}

Channel capacity $\mathrm{C}=15$

Total number of patients $=87$.

Total inter-arrival time $=781$ minutes.

Total service time $=658$ minutes

By (7), $\lambda=6.6837 / \mathrm{hr}$; by (8) $\mu=7.9331 / \mathrm{hr}$; by (9), $\rho=0.8425$

\section{Category $(D)$}

Channel capacity $\mathrm{C}=15$

Total number of patients $=87$

Total inter-arrival time $=1013$ minutes.

Total service time $=701$ minutes

By (7), $\lambda=5.1530 / \mathrm{hr}$; by (8), $\mu=7.4465 / \mathrm{hr}$; by (9), $\rho=0.6920$

\subsubsection{Results with trial model}

The following results were obtained concerning queueing time and service time as shown in the table below:

By (14) and (15) respectively, we have

\begin{tabular}{lrrrr} 
Variable & Staff & \multicolumn{4}{c}{ Students } & Gynaecology Private \\
Mean time in system & 37.35 & 18.28 & 38.61 & 25.68 \\
Mean time on queue & 29.54 & 9.93 & 30.95 & 17.62
\end{tabular}

A comparison of all these results shows that time spent in the system and on queue in all the categories is still very large indicating that this model has failed to produce the desired result of eliminating or reducing queue time to the barest minimum. There is therefore the need to construct another model. This time the categories are retained but with just one queue as shown in the proposed model below.

\subsection{The Proposed Model}

To formulate a suitable model with characteristics that will enable a solution to this problem of long wait be achieved, a modification of the original model in the area of number of channels was made thus applying the principle of $(\mathrm{m} / \mathrm{m} / \mathrm{k})$ the multi-channel system. On analysis, this model proved workable as it produced the desired result of reducing queueing time. It is therefore presented here as the proposed model.

\subsubsection{M/M/4/FCFS $/ \infty / \infty$}

This is a multy-channel queueing model with 4 channels, arrival and service times are both exponential. The queue discipline is first come first serve. It has one queue from which patients are allocated to the channels.

\subsubsection{Computations with Proposed Model}

After analyzing data, computations and results were as follows:

Total number of patients $=350$

Number of channels (doctors) $\mathrm{K}=4$

Total arrival time $=1146$ minutes.

Total service time $=2764$ minutes .

Mean arrival rate $\lambda=\frac{\text { total no.of patients }}{\text { total arrival time }}=\frac{350}{1146}=0.3054$ 
Mean service rate $\mu=\frac{\text { total no.of patients }}{\text { total service time }}=\frac{350}{2764}=0.1266$

The ratio $\frac{\lambda}{\mu}=\frac{0.3054}{0.1266}=2.4123$

Service intensity $\rho$ by $(1)=0.6031$

The probability of no patient in the hospital $\mathrm{P}_{0}$ by $(6)=0.0819$.

Here the mean queue length $\mathrm{Lq}$ by $(2)=0.4422 \approx 0.44$ patients

Mean time spent on queue Wq by $(4)=1.4484 \approx 1$ minute

Mean number of patients in the Hospital L by $(3)=2.8545 \approx 3$ patients

Mean time spent in the Hospital $\mathrm{W}$ by $(5)=9.3468 \approx 9$ minutes

It is obvious that this model provides a better result to this queueing problem as the queue disappears and the waiting time reduces to minimum with the model. With this model a patient spends an average time of 9 minutes in the hospital and just one minute in the queue. Results further show that there is an average of 0.44 persons in the queue.

It is clearly seen from the above results that patients spend little or no time in the queue to consult a doctor. In fact a queue length of 0.44 shows that the queue has disappeared, and waiting time reduced to the barest minimum.

The application of this model however, implies the addition of another consultant which invariably involves additional cost, but the benefit derivable by this particularly outweighs this cost.

\section{Conclusion}

This paper analyzed the existing structure then went ahead to formulate a model which on application with system characteristics was found workable. In fact a comparison of results on application of the models showed that while queue persisted in the existing and trial models and patients spent much time on queue, the queue disappeared in the proposed model and waiting time reduced drastically. The advantage of the proposed model lies in its capacity to reduce waiting time. Most importantly, it provides a tool to assist management of the polytechnic health centre and the nation at large take decision as to the number of facilities to provide in hospitals to avert delays Since the experience in the polytechnic health centre is a replica of what happens in the health centres nationwide.

\subsection{Recommendations}

In tandem with results obtained from analytical investigations, it is recommended that:

- Shehu Mohammad Kangiwa Medical Center and other medical centres in the country at large adopt the proposed model as it has been proven to be workable and has the capacity to drastically reduce the time spent on queues

- More medical doctors be employed in Shehu Mohammad Kangiwa Medical center in particular and other medical centers or hospitals in the country at large where there are queueing problems.

\section{References}

[1] Wagner .H.M principle of management science

[2] Russell 1. Ackoff and Maurice w. Sasieni fundamentals of operations research. (Wiley Easter ltd ,1986)

[3] Frederick S.H. and Gerald J.L (2007), Introduction to Operations Research. Concept and Cases $8^{\text {th }}$ Edition. Tata (Mcgraw-Hill Publishing Company Ltd. New Delhi ,2007).

[4] Kothary C,R An Introduction to Operations Research, (Vikas Publishing House, New Delhi ,2008).

[5] Ivo Adan and Jaques Resing queueing theory department of maths \& comp. sc Eudhoven university of technology Netherlands (2002)

[6] Cheema D.S operations research laxmi publications Ltd New Delhi (2006)

[7] Oladejo unpublished lecture notes Nigerian Defence Academy, Nigeria (2010)

[8] Dombacher C. queueing models for call centres (2010) Nikolaus lenaugasse 8, A-2232 Deutsch-Wagram.

[9] Sang $\mathrm{m}$. lee and etal introduction to decision science (1975)

[10] Raj Jain introduction to queueing theory (2008), http://www.csc.wush.edu/.jain/csc567-08 